\title{
Midterm Survival of a Varus-Valgus Constrained Implant following One-Stage Revision for Periprosthetic Joint Infection: A Single-Center Study
}

\author{
Baochao Ji, MD, PhD ${ }^{1, *}$ Guoqing Li, MD ${ }^{1, *}$ Xiaogang Zhang, MD ${ }^{1} \quad$ Yang Wang, MD ${ }^{1}$ \\ Wenbo Mu, MD, PhD ${ }^{1}$ Li Cao, MD, FRCS ${ }^{1}$ \\ ${ }^{1}$ Department of Orthopaedics, First Affiliated Hospital of Xinjiang \\ Medical University, Urumqi, Xinjiang, China \\ Address for correspondence Li Cao, MD, FRCS, Department of \\ Orthopaedics, First Affiliated Hospital of Xinjiang Medical University, \\ J Knee Surg 2023;36:284-291. \\ 137 South LiYuShan Road, Urumqi, Xinjiang 830054, China \\ (e-mail: xjbone@sina.com).
}

\begin{abstract}
Keywords

- varus-valgus constrained Implant

- one-stage revision

- total knee arthroplasty

- periprosthetic joint infection
\end{abstract}

Revision total knee arthroplasty (TKA) is challenging to perform in patients with periprosthetic joint infection (PJI) due to the difficulty of eradicating the infection and potential for bone and ligamentous deficits. The current study aimed to evaluate the midterm survival of varus-valgus constrained (VVC) implants used in one-stage revision TKA for chronic PJI at our institution. This retrospective analysis included 132 patients with chronic PJI who underwent one-stage revision using a VVC implant. The average follow-up was 51.6 months (range: 24-85 months). Five-year survival analysis was performed to set recurrent infection and mechanical failure as the end point. Hospital for special surgery (HSS) as functional outcomes was evaluated preoperatively and at the latest follow-up. A total of 12 patients $(9.1 \%)$ underwent retreatment for reinfection (nine patients) and mechanical failure (three patients). The overall 5 -year revision-free survival was $82.7 \%$, the infection-free survival was $91.1 \%$, and the mechanical failure-free survival was $98.3 \%$. The preoperative HSS knee score improved from 35.6 points (range: $24.3-47.7$ points) preoperatively to 76.8 points (range: 57.2-87.6 points) at the latest follow-up. Complications were identified in 20 patients (15.2\%) which included aseptic osteolysis in 4 cases, acceptable flexion instability in 3 cases, arthrofibrosis in 2 patients, hematomas in 2 cases, calf intermuscular venous thrombosis in 6 patients, and femoral stem tip pain in 3 cases. This is the first study to report the outcomes of one-stage revision using VVC implants for knee PJI. Improved functional outcomes and good midterm survival are demonstrated at an average follow-up of 51.6 months.
The treatment of periprosthetic joint infection (PJI) is associated with not only tremendous medical efforts but also high health care costs. ${ }^{1}$ The incidence of infection following primary total knee arthroplasty (TKA) ranges from 0.4 to $4.0 \%, 2,3$ and the incidence will steadily increase as the

\footnotetext{
These authors contributed equally to the work described in the article and share first-author status.
}

received

January 18, 2021

accepted after revision

May 31, 2021

article published online

July 16, 2021

demand for primary TKA continues to increase globally. Indeed, the projections based on U.S. Medicare data demonstrate that the number of primary TKA procedures performed is expected to increase by $673 \%$ by $2030 .^{4}$

Revision TKA in patients with PJI is a complex procedure due to the difficulty of eradicating the infection and potential for bone and ligamentous deficits. Once chronic PJI has been identified, whether one-stage or two-stage revision is

(c) 2021. Thieme. All rights reserved. Thieme Medical Publishers, Inc., 333 Seventh Avenue, 18th Floor, New York, NY 10001, USA
DOI https://doi.org/ 10.1055/s-0041-1731739. ISSN 1538-8506. 
performed, the mandatory principle is aggressive eradication of all organic and nonorganic material that is dead or infected in the first step. Therefore, different degrees of ligament deficiency are inevitable in most patients after intraoperative debridement. One method of overcoming this challenge is to increase the constraints of the implant. Depending on the degree of ligamentous laxity present, various types of prostheses have been proposed over the years, including range from posterior stabilized (PS) prostheses, varus-valgus constrained (VVC) implants to hingetype prostheses.

A VVC knee prosthesis was first designed in 1977 which consists of a highly constrained unlinked prosthesis with a broad elevated tibial post that articulates with a deep femoral box, providing increased medial-lateral stabilization. ${ }^{5}$ The constraint of this design is more than PS and less than hinge prostheses that cannot only provide reliable stability in most revision knee arthroplasties but also reduce the risk of mechanical failure due to excessive constraints and save the host bone.

Over the past few decades, many studies on VVC prostheses have been published ${ }^{6,7}$; however, to the best of our knowledge, there are no reports on the survival of VVC implants following one-stage revision for infection. Given that the one-stage revision is receiving increasing attention due to low mortality, good functional outcomes, and high satisfaction, ${ }^{8,9}$ this information is much needed to aid the selection of implants for onestage knee revision for infection.

Therefore, the purpose of this study was to evaluate the midterm survival of VVC implants used in revision TKA for PJI at our institution and determine (1) the infection-free survival; (2) the mechanical failure-free survival; (3) the clinical outcome, as measured by the hospital for special surgery (HSS) score ${ }^{10}$; and (4) the postoperative complications.

\section{Materials and Methods}

\section{Patient Demographics}

This study was approved by the institutional review board. Using the data in the registry of our institution, which were entered prospectively, we identified 160 patients (160 knees) who had undergone one-stage revision TKA in the presence of PJI between January 2011 and June 2018. Our exclusion criteria for single-stage revision are according to the system by McPherson et al. ${ }^{11}$ The single-stage revision was basically not performed on severely immune-compromised patients or patients with active systemic infection or with severely limited cardiovascular function and were unlikely to tolerate any surgery. For local joint status, our contraindications of single-stage revision included the following: (1) extensive periarticular soft-tissue damage, so that joint function cannot be reconstructed by revision operation; (2) patients with wide infection disseminate to other cavities, such that infection cannot be eradicated completely; and (3) infection involving the neurovascular bundles as well. Beside these exclusion criteria, fungal infection, culture-negative PJI, and multidrug resistant bacteria are all included as our inclusion criteria of one-stage
Table 1 Demographic characteristics of the 132 patients with infected TKA who underwent single-stage revision with VVC implants

\begin{tabular}{|c|c|}
\hline Variable & $\begin{array}{l}\text { Value } \\
\text { Mean (range) or } n(\%)\end{array}$ \\
\hline Male:female & $34: 98$ \\
\hline Mean age (y) & $68.6(45-83)$ \\
\hline BMI $\left(\mathrm{kg} / \mathrm{m}^{2}\right)$ & $26.3(18.6-38.4)$ \\
\hline Hypertension & $57(43.2)$ \\
\hline Diabetes mellitus & $30(22.7)$ \\
\hline Rheumatoid arthritis & $3(2.3)$ \\
\hline Other comorbidities & $14(10.6)$ \\
\hline Sinus tract & $37(28.0)$ \\
\hline $\begin{array}{l}\text { Preoperative CRP }(95 \% \mathrm{Cl}) \text { in } \\
\mathrm{mg} / \mathrm{L}\end{array}$ & $32.4(17.4-61.1)$ \\
\hline $\begin{array}{l}\text { Preoperative ESR }(95 \% \mathrm{CI}) \text { in } \\
\mathrm{mm} / 1 \text { hour }\end{array}$ & $54.1(42.3-71)$ \\
\hline $\begin{array}{l}\text { Mean total leucocyte count } \\
\left(\mathrm{mm}^{3}\right)\end{array}$ & $6,551(4,880-9,210)$ \\
\hline $\begin{array}{l}\text { Mean duration of symptoms } \\
\text { prior to single-stage revision } \\
\text { in months (range) }\end{array}$ & $10.3(6-21)$ \\
\hline \multicolumn{2}{|l|}{ Pathology } \\
\hline Neutrophils $\geq 10 / \mathrm{HPF}$ & $132(100)$ \\
\hline
\end{tabular}

Abbreviations: BMI, body mass index; CRP, C-reactive protein; $\mathrm{Cl}$, confidence interval; ESR, erythrocyte sedimentation rate; HPF, high power field at $\times 400$ magnification; TKA, total knee arthroplasty; VVC, varus-valgus constrained.

revision. ${ }^{12}$ Eleven of 160 patients were excluded because the VVC prosthesis was not used for the revision; instead, eight PS implants and tibia stems, two rotating-hinge knees, and one tumor prosthesis were used. Data on 149 patients were entered into the database. However, 12 of the 149 patients were lost to follow-up within 2 years, and 5 patients died of unrelated causes during the study period. Finally, only 132 patients (132 knees) were included in the study. There were 34 males and 98 females. The average age was 68.6 years (range: 40-86 years) at the time of the index revision surgery. The demographic characteristics and a description of the cohort are presented in - Table $\mathbf{1}$.

\section{Diagnosis of Periprosthetic Joint Infection}

Patients were definitively diagnosed with infected TKA on the basis of the Musculoskeletal Infection Society criteria. ${ }^{13}$ Joint aspiration was performed preoperatively in all patients. Antibiotic therapy was discontinued strictly for a minimum of 2 weeks before aspiration. The duration of culture was extended from 5 to 21 days, depending on the culture results. The pathogen culture protocols are described in previous study. ${ }^{14}$

\section{Surgical Technique}

All one-stage revision procedures were performed in accordance with the same standardized surgical protocol by the 
same senior surgeon who is experienced in knee revision and infection treatment. Pathogen-sensitive antibiotics were administered intravenously 30 minutes before an incision was made. We divided our technique into three distinct stages:

\section{Aggressive Debridement}

The previous skin scar was excised, and the fistulae were integrated into the skin incision, if possible, and radically excised all the way to the joint. Debridement was performed without blood evacuation, so that the boundaries between the infected tissue, scar, and healthy bleeding soft tissue (and bone) could be distinguished better during the process. This step involved the removal of all bone sequestra and proliferative inflammatory synovium, including that in the medial and lateral gutter areas and the suprapatellar pouch by using a rongeur and a curette. The debridement performed on collateral ligaments should be meticulous. On the basis of thorough debridement, the damage of the medial and lateral collateral ligament should be minimized. A visible fresh soft tissue plane with clear boundaries was visible after debridement. In addition, several samples (at least three) were acquired from the areas with the most florid inflammatory changes for culture, sensitivity, and histological tests. Afterwards, the components and cement debris were removed thoroughly using specific devices. After explantation, inflammatory tissue and biofilm on the bone-prosthesis interface and intramedullary canal were cleared using a burr and curette. Fresh bone beds with errhysis are the standard for debridement, and bone defects after debridement are often considered second. The surgical area was then extensively irrigated manually with at least 3 to $10 \mathrm{~L}$ of saline and 100 to $200 \mathrm{~mL}$ of a $3 \%$ hydrogen peroxide solution. The wound was then provisionally closed and the surgical field was soaked in $0.5 \%$ of aqueous betadine for 15 minutes.

\section{Intermission}

The surgical area was then resterilized and redraped, and the surgical team rescrubbed. The surgical gowns and the entire set of surgical instruments were also replaced.

\section{New Prosthesis Implantation}

After another round of wound cleaning by pulsed lavage with 3-L $0.9 \%$ saline, implants were chosen based on the stability and the size of the bony defects that were classified and recorded according to the Anderson Orthopaedic Research Institute (AORI) bone defect classification system. ${ }^{15}$ The stability of knee joints was evaluated with the PS trial. Then, the VVC prosthesis rather than the PS prosthesis was selected when the surgeon encountered one of the following situations: an asymmetrical extension and flexion gap, an uncorrectable large flexion gap, midflexion instability, or posterolateral subluxation. The use of increased constraints without stems was rarely indicated and was restricted to cases of severe soft tissue laxity associated with reliable metaphyseal bone quality. Most stems were fixed using the cementing technique, and some were fixed using the hybrid cementing technique. ${ }^{16}$ Although the stems with the hybrid pattern was not fully cemented, the metaphyseal portions that exceeded the Morse taper were cemented. The length of the stem varied according to the degree of the tibial/femoral deformity, bone quality, and presence of concomitant proximal femoral components. Vancomycin powder of $1 \mathrm{~g}$ was poured into the femoral and tibia canal before the implantation of a new prosthesis. All infected knees received a cemented prosthesis. Then, another $0.5 \mathrm{~g}$ of vancomycin powder was poured into the whole joint cavity before the capsule was closed.

\section{Postoperative Antibiotic Treatment Strategies and Rehabilitation}

All individual antibiotic treatments were prescribed by the treating surgeon in consensus with the local department of clinical microbiology. The strategies of patients with fungal infection and positive and negative cultures employed have been presented in previous studies. ${ }^{12,14,17}$ Active range of motion exercises performed in bed and weight bearing as tolerated were started on postoperative day 1 . All of the patients were allowed to stand at the bedside at the beginning. To avoid excessive exudation in the joint, walking with crutches was not recommended until approximately 2 weeks after the operation.

\section{Follow-up}

The patients were routinely followed up in the outpatient clinic at 1,3, and 6 months postoperatively and then annually thereafter to assess the serum erythrocyte sedimentation rate (ESR) and C-reactive protein (CRP) level for infection and the HSS knee score for function. Plain radiographs (full-leg length and anteroposterior and lateral views) were taken in the radiological examinations and evaluated by two observers who were not involved with the surgery; they assessed the presence and location of all radiolucent lines at the cement-bone or cement-implant interface according to the recommendations of Insall et al. ${ }^{18}$ For patients who cannot complete follow-up at clinic, they were advised to have the radiographs taken at the local hospital and post to our hospital.

\section{Survival Analysis and Outcome Measures}

The primary endpoint included infection-free survival at the 5-year follow-up. Rerevision surgery for mechanical failure (unrelated to PJI or sepsis) was the second endpoint for our analysis. Mechanical failure was defined as the loss of function of the prosthesis and/or unfavorable relationships between the prosthetic components and adjacent bone and soft tissue attachments.

The infection was considered treated to a satisfactory extent when there were no clinical symptoms or signs of infection at the last follow-up. Treatment failure was defined as the recurrence of an infection in the same knee.

\section{Statistical Analysis}

Fisher's exact test was used to compare the distribution frequencies of the categorical variables. Mann-Whitney $U$ tests were performed to examine the differences between 
the preoperative and postoperative HSS scores. The VVC implant survivorship and infection-free survivorship were estimated using Kaplan-Meier survival analysis. The statistical tests were performed using SPSS software (SPSS Inc., Chicago, IL), with $p$-values of $<0.05$ indicating statistical significance.

\section{Results}

A total of 12 patients (9.1\%) had undergone additional treatment of the index knee joint at a mean of 51.6 months of follow-up (range: 24-85 months). Kaplan-Meier survival analysis demonstrated that the overall 5-year survival was 82.7\% (95\% confidence interval [CI]: 68.7-96.8\%; - Fig. 1) and that the estimated 5 -year survival using recurrent infection as the end point was $91.1 \%$ (95\% CI: 85.3-96.9\%; -Fig. 2). When mechanical failure was used as the endpoint, the 5year survival was 98.3\% (95\% CI: 95.9-99.8\%; - Fig. 3). Information of the implants is presented in - Table $\mathbf{2}$. On femoral side, there were 31 (23.5\%) cases reconstructed by augments

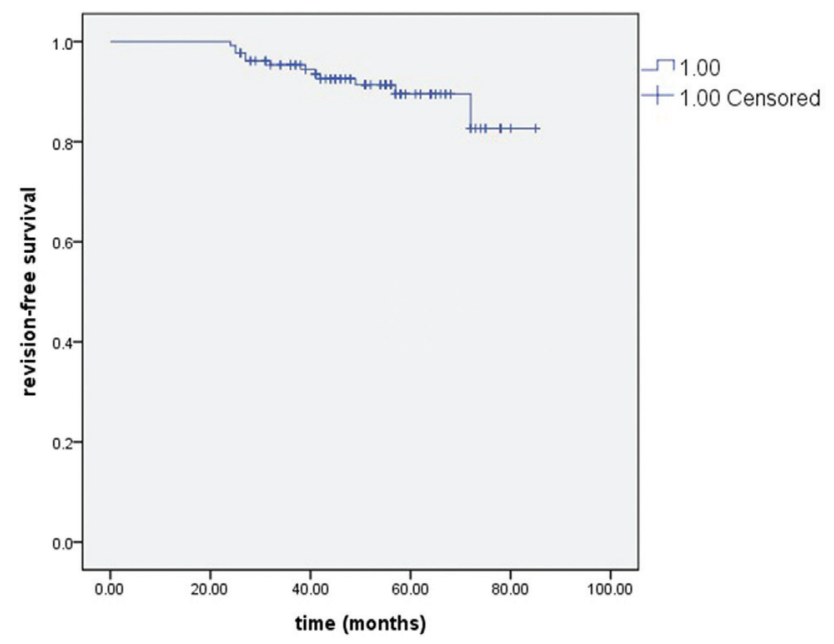

Fig. 1 Overall survival of the varus-valgus constrained implants by Kaplan-Meier survival analysis.

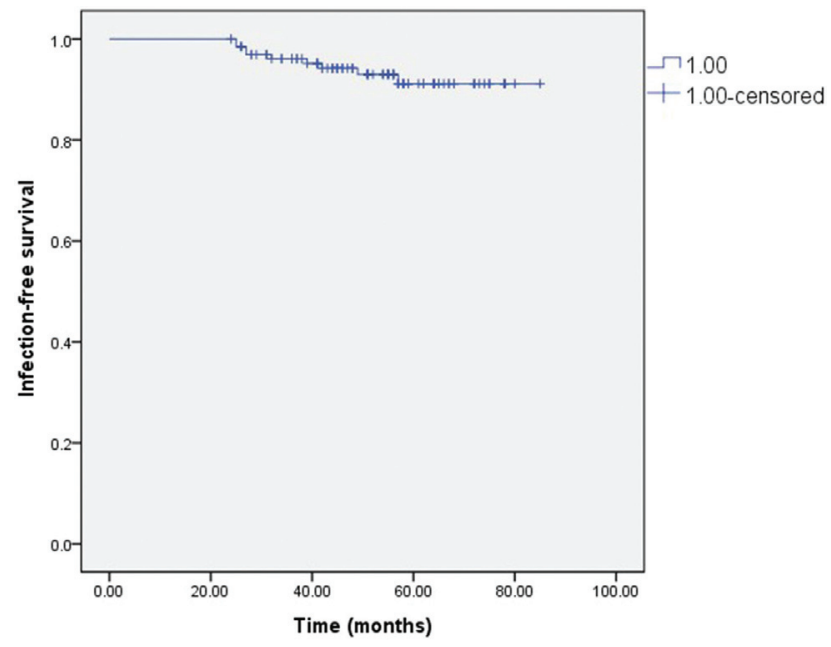

Fig. 2 Infection-free survival of varus-valgus constrained implants by Kaplan-Meier survival analysis.

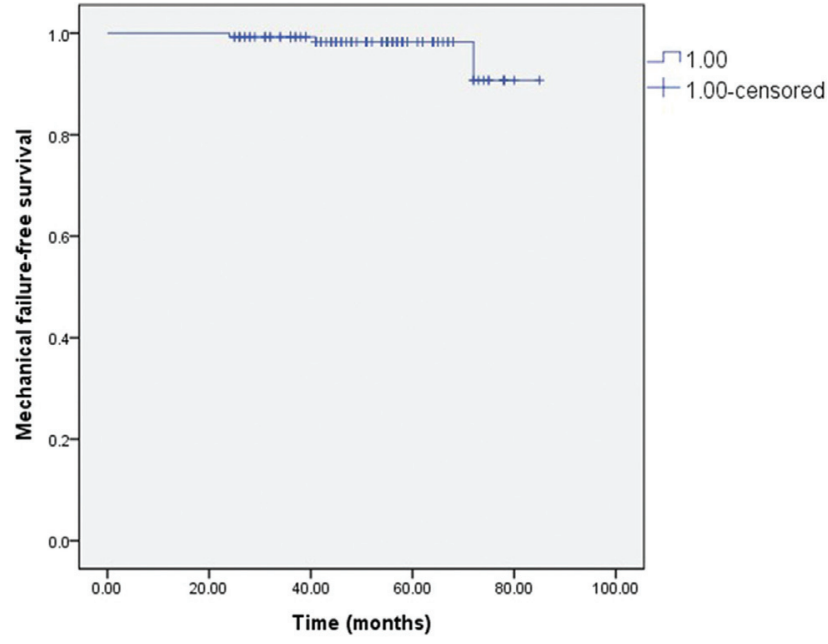

Fig. 3 Mechanical failure-free survival of varus-valgus constrained implants by Kaplan-Meier survival analysis.

Table 2 Information on the implants

\begin{tabular}{|l|l|}
\hline Implants & $\boldsymbol{n}(\%)$ \\
\hline $\begin{array}{l}\text { NexGen LCCK (fixed-bearing; Zimmer } \\
\text { Biomet, Inc., Warsaw, IN) }\end{array}$ & $95(71.9)$ \\
\hline $\begin{array}{l}\text { Legion Revision System (fixed-bearing; } \\
\text { Smith \& Nephew, London, United } \\
\text { Kingdom) }\end{array}$ & $33(25)$ \\
\hline $\begin{array}{l}\text { P.F.C Sigma (mobile-bearing; DePuy, } \\
\text { Warsaw, IN) }\end{array}$ & $4(3.0)$ \\
\hline $\begin{array}{l}\text { Fixation of the femoral component and } \\
\text { stem }\end{array}$ & \\
\hline Cemented & $107(69.7)$ \\
\hline Uncemented & $0(0.0)$ \\
\hline Hybrid & $25(18.9)$ \\
\hline $\begin{array}{l}\text { Extensive stem used on the femoral } \\
\text { and tibial sides }\end{array}$ & $132(100.0)$ \\
\hline $\begin{array}{l}\text { Mean polyethylene thickness (mm) } \\
\text { Mean (range) }\end{array}$ & $13.5(10-18)$ \\
\hline Reinforced devices & \\
\hline Femoral & $6(4.5)$ \\
\hline Augments & $36(27.3)$ \\
\hline Cone & $15(11.4)$ \\
\hline Cone + augments & $2(1.5)$ \\
\hline Sleeve & $1(0.8)$ \\
\hline Sleeve + augments & $19(14.4)$ \\
\hline Tibial & $3(2.3)$ \\
\hline Autograft & $1(0.8)$ \\
\hline Augments & \\
\hline Cone & \\
\hline Cone + augments & \\
\hline Sleeve & \\
\hline
\end{tabular}


and 19 (14.4\%) patients reconstructed by cone. The sleeves were used in four knees. On tibia side, the cones were chosen in $15(11.4 \%)$ cases and the sleeve was implanted in one patient. According to the Anderson Orthopaedic Research Institute (AORI) classification system, the femoral bone defects were as follows: 56 were type I, 26 were type IIA, 29 were type IIB, and 21 were type III. A total of 58 type I, 35 type IIA, 23 type IIB, and 16 type III defects were on the tibial side.

\section{Septic Failure}

Nine patients of the 132 (6.8\%) one-stage revision knees for chronic infection were reinfected based on the clinical parameters and serologic measures at a mean follow-up of 51.6 months (range: $24-85$ months). The mean value of ESR and CRP at the time of their infection recurrence was $57.1 \mathrm{~mm} / \mathrm{h}$ (range: $40-68 \mathrm{~mm} / \mathrm{h}$ ) and $29.5 \mathrm{mg} / \mathrm{L}$ (rage: $10.3-44.2 \mathrm{mg} / \mathrm{L})$, respectively. The average time from the index revision procedure to reinfection was 35.9 months (range: 25-57 months). Repeat surgeries for reinfection were performed in seven knees that was the most common cause of reoperation in VVC implants in current study, including four aggressive debridements with retained components, one one-stage rerevision, and two above-knee amputations. One of the patients who underwent amputation had uncontrolled rheumatoid arthritis, diabetes mellitus, and a multidrug-resistant infection with methicillin-resistant Staphylococcus epidermidis (MRSE). Moreover, the patient developed a periprosthetic femoral fracture and an unmanageable large sinus tract due to a fall at home. The patients did not agree to undergo salvage surgery because of the psychological and financial burden. Another patient who underwent amputation had uncontrolled rheumatoid arthritis and previously underwent four debridement and one spacer implantation procedures. The patient had sinus tract communication with the prosthesis at 25 months after the one-stage revision and had a polymicrobial infection involving Candida albicans and Staphylococcus aureus. Because the patient had a severe soft tissue condition and an extremely high financial burden, the patient agreed to undergo amputation. Additionally, two patients with an immunocompromised status refused additional operations and accepted only long-term chronic suppressive antibiotic therapy. Twenty-seven patients with culture-negative infected knees were included in the current study, and 2 of them exhibited relapse at the last follow-up.

Three of the nine failure cases were fungal infections. Gram-positive bacteria were the most common pathogens $(74 / 132,56.1 \%)$ in the current study. The microbiological findings are listed in -Table 3.

\section{Mechanical Failure}

There were three patients (2.3\%) underwent rerevised for mechanical failure. One implant was replaced with another LCCK implant with a longer stem due to ligamentous instability that led to repeated dislocation of the insert at the 7month follow-up. The patient with a stable knee was followed-up at the clinic 47 months of postoperation. Two knees sustained a supracondylar periprosthetic femur fracture, which required revision of the femoral component only. The metaphyseal sleeves and cables were used for the typeIII bone defects.

\section{Clinical Outcomes}

Ninety-eight patients (74.2\%) were meet in the office for complete clinical and radiographic assessment at a mean of 46.5 months of follow-up (range: 24-61 months) and 34 patients $(25.8 \%)$ only had a telephone evaluation for the HSS score at an average of 53.9 months of follow-up (range: 2485 months) and taken radiographs in their local hospital. The HSS assessment therefore administered to all included patients at the most recent follow-up demonstrated significant improvement, from a mean preoperative score of 35.6 (range: $24.3-47.7$ ) to 78.2 (range: $59.3-88.9$ ) points at a minimum of 1 -year follow-up ( $p<0.05$ ), and with no significant deterioration noted at the final follow-up (mean HSS score: 76.8 points (range: $57.2-87.6$ points).

\section{Complications}

Complications were identified in 20 patients (15.2\%). There were no cases of complete radiolucent lines (RLLs) at the final follow-up. Four cases (3.0\%) with aseptic osteolysis at the medial tibia after a mean of 58 months of follow-up (range: 51-62 months) were not progressive, and no revisions were subsequently performed. Three cases (2.3\%) with acceptable flexion instability were observed after a mean follow-up of 42 months (range: 34-53 months). Since these patients were asymptomatic and had a functional prosthesis, a close follow-up without surgical intervention was performed during the study period. In addition, we noticed two patients (1.5\%) who underwent a manipulation under anesthesia for arthrofibrosis and two hematomas (1.5\%). Calf intermuscular venous thrombosis was reported in six $(4.5 \%)$ of the patients during the perioperative period. The ankle dorsiflexion exercise was intensified after short-term rivaroxaban was given to the patients. These thrombosis cases were considered to have a reperfusion status at a mean of 21 days after the operation. Three patients $(2.3 \%)$ had femoral stem tip pain, and no patients had tibial stem tip pain during weight bearing. The degree of stem tip pain was mild in all patients and did not require another revision.

\section{Discussion}

When revision TKA is performed, the prosthesis with the fewest constraints is preferred because a large number of constraints might lead to mechanical loosening and prosthesis failure. ${ }^{19}$ However, there are instances where less-constrained prostheses are not able to generate the required stability which inevitably forces surgeons to choose a more constrained prosthesis. Innovations in the implants designed for revision TKA have provided surgeons with many alternatives to address such difficult challenges. The VVC prosthesis system was designed to have more constraints than the PS but fewer constraints than the hinge implant. Compared with the number of studies on the outcomes and 
Table 3 Microbiologic findings and postoperative antibiotic treatment regimens

\begin{tabular}{|c|c|c|c|c|c|}
\hline Microorganism & $\begin{array}{l}\text { No. (\%) of episodes } \\
n(\%)\end{array}$ & $\begin{array}{l}\text { Initial IV } \\
\text { antibiotics }\end{array}$ & $\begin{array}{l}\text { Intra-articular } \\
\text { antibiotics } \\
\text { infusion }\end{array}$ & $\begin{array}{l}\text { Oral switch } \\
\text { antibiotics }\end{array}$ & $\begin{array}{l}\text { Outcome, } \\
\text { number }\end{array}$ \\
\hline Gram-positive bacteria & $74(56.1)$ & & & & \\
\hline Staphylococcus aureus & $21(15.9)$ & VAN & None & LVFX + RFP & One relapse \\
\hline Staphylococcus epidermidis & $19(14.4)$ & VAN & None & LVFX + RFP & No relapse \\
\hline MRSE & $14(10.6)$ & VAN & VAN & LVFX + RFP & One relapse \\
\hline MRSA & $11(8.3)$ & VAN & VAN & LVFX + RFP & One relapse \\
\hline Staphylococcus lentus & $2(1.5)$ & VAN & None & LVFX + RFP & No relapse \\
\hline Enterococcus fecalis & $2(1.5)$ & VAN & None & LVFX + RFP & No relapse \\
\hline other & $5(3.8)$ & VAN & None & LVFX + RFP & No relapse \\
\hline Gram-negative bacteria & 25 (18.9) & & & & \\
\hline Klebsiella pneumoniae & $9(6.8)$ & MER & None & LVFX + RFP & One relapse \\
\hline Escherichia coli & $6(4.5)$ & IMI & None & LVFX + RFP & No relapse \\
\hline Enterobacter cloacae & $4(3.0)$ & IMI & None & LVFX + RFP & No relapse \\
\hline Pseudomonas aeruginosa & $3(2.3)$ & IMI & None & LVFX + RFP & No relapse \\
\hline Proteus mirabilis & $2(1.5)$ & IMI & None & LVFX + RFP & No relapse \\
\hline Acinetobacter baumannii & $1(0.8)$ & IMI & None & LVFX + RFP & No relapse \\
\hline Culture-negative results & $27(20.5)$ & VAN & VAN + IMI & LVFX + RFP & Two relapses \\
\hline Fungus & $5(3.8)$ & & & & \\
\hline Candida glabrata & $2(1.5)$ & $\mathrm{VAN}+\mathrm{FLU}$ & FLU & $\mathrm{LVFX}+\mathrm{RFP}+\mathrm{FLU}$ & One relapse \\
\hline Candida parapsilosis & $2(1.5)$ & $\mathrm{VAN}+\mathrm{FLU}$ & FLU & $\mathrm{LVFX}+\mathrm{RFP}+\mathrm{FLU}$ & One relapse \\
\hline Aspergillus & $1(0.8)$ & VAN + VOR & VOR & $\mathrm{LVFX}+\mathrm{RFP}+\mathrm{ITR}$ & One relapse \\
\hline Polymicrobial infections & $1(0.8)$ & & & & \\
\hline S. epidermidis, S. lentus, E. coli & $1(0.8)$ & IMI & VAN + IMI & LVFX + RFP & No relapse \\
\hline Total & $132(100)$ & & & & Nine relapses \\
\hline
\end{tabular}

Abbreviations: FLU, fluconazole; IMI, imipenem; ITR, itraconazole; IV, intravenous; LVFX, Levofloxacin; MER, meropenem; MRSA, methicillin-resistant Staphylococcus aureus; MRSE, methicillin-resistant Staphylococcus epidermidis; RFP, rifampicin; VAN, vancomycin; VOR, voriconazole.

survival of VVC prostheses in complex primary TKA and aseptic revision, ${ }^{20,21}$ there is a paucity of studies reporting the survival of VVC implants following revision for infection. To our knowledge, only one study reported the outcomes of two-stage revision, ${ }^{22}$ and no studies reported the outcomes of one-stage revision. Because these two approaches involve different techniques, data on one-stage knee revision are much needed to help implant selection. Our intent was to determine the outcomes of one-stage revision TKA with a VVC system in the presence of a PJI. We specifically examined infection-free survival, mechanical failure-free survival, functional improvement as measured by the HSS score, and complications.

When infection-free survival was used as the endpoint, the survival was $91.1 \%$ at 5 years; this result was comparable to that reported by Pangaud et $\mathrm{al}^{23}$ in a systematic review of oneand two-stage revisions for infected TKA. The average eradication rate was $87.1 \%$ for the one-stage procedure and $84.8 \%$ for the two-stage procedure. However, the patient inclusion criteria for the single-stage procedure were broader in the current study which included patients with culture-negative results, multidrug-resistant organisms, and fungal infections. According to the previous data, with this broad inclusion criteria associated with infected total hip arthroplasty, the infection control rate was $89.2 \%$ at a mean follow-up of 58 months which was encouraging. ${ }^{12,17}$ Defining the eligibility criteria for one-stage revision is an ongoing challenge. There are no published randomized controlled trials (RCTs) that provide concrete evidence regarding the effectiveness of traditional inclusion or exclusion criteria. Jenny et $\mathrm{al}^{24}$ described a similar infection-free survival rate after 4 years in patients who underwent routine one-stage revision (85\%) versus selected patients with infection who underwent TKA (78\%). Massin et $\mathrm{al}^{25}$ recently reported factors contributing to recurrent infection after two-stage and routine one-stage total knee revisions. The presence of multidrug-resistant bacteria, fistula, and culture-negative infection were analyzed by univariate and multivariate logistic regression analyses. The authors' results indicated that the type of surgery was not significantly correlated with recurrent infection.

Three patients $(2.3 \%)$ had to undergo another surgical intervention due to mechanical failure after our one-stage 
revision. The survival rates of the prostheses in this study were better than those in earlier reports and were comparable to those in recent reports. The failure rate for the VVC prosthesis used in the primary TKAs ranged from 0 to $22 \%, 20,26,27$ and the rate ranged from 0 to $35.7 \%$ for revisions. ${ }^{22,27,28}$ In the only study that has reported the outcomes of two-stage revision using the VVC implant, Wilke et $\mathrm{al}^{22}$ reported a case with $71 \%$ overall 5 -year survival and an estimated 64\% 10-year survival when repeat revision surgery was used as the end point. When recurrent infection was used as the endpoint, the 5-year survival was $77 \%$, and the 10 -year survival was $68 \%$. Furthermore, in the largest study conducted to date, Siqueira et $\mathrm{al}^{27}$ included 685 consecutive cases with VVC implants. The results showed that the 10 year survivorship was 80.1 and $85.3 \%$ for aseptic and septic revisions, respectively. When failure for any reason was considered as the end point, compared with aseptic revision, septic revision had 2.1 times higher risk of failure. Moreover, in the study with the longest follow-up period, Kim YH et $\mathrm{al}^{28}$ revealed that the 16-year rate of survival of the VVC components was $94.7 \%$ with loosening as the end point and $92 \%$ with revision as the end point.

The VVC designs with a broad elevated tibial post that articulate with a deep femoral box may provide satisfactory varus-valgus support for most patients and have the advantage of allowing the center of rotation to change during flexion, thereby theoretically imparting less tangential anteroposterior stress across the prosthetic interface. However, fixed-bearing VVC knees are more rotationally constrained than are rotating-hinge designs and theoretically impart greater rotational stresses to the fixation interfaces. Therefore, it is critical that the femoral and tibial components are aligned properly in the rotational direction during revisions to avoid early loosening and fracture of the intercondylar post. It is noted that four VVC knees with mobile bearing TKA were used in current study which were performed in recent years. Mobile bearings were introduced in an attempt to reduce polyethylene wear and possibly reduce torque at the bone-implant interface. Although the benefit for the use of mobile-bearing compared with fixed-bearing TKA is still controversial, ${ }^{29-31}$ a mobile-bearing VVC theoretically provides less rotational stresses to the fixation interfaces and offers greater tolerance to rotational malalignment between femoral and tibial components that may be a reasonable option in revision TKA. Moreover, VVC prostheses do not prevent hyperextension. Progressive hyperextension may result when there is muscular weakness or the absence of posterior capsular and muscular attachments secondary to massive bone loss, segmental resection, or a neuromuscular disease such as Charcot's arthropathy. In these situations, a rotating-hinge design with a mechanical stop for hyperextension may be a more appropriate choice.

The mean HSS score in the current study increased significantly to 78.2 at a minimum follow-up of 1 year, and it remained the same at the time of the final follow-up. The magnitude of improvement was comparable to that observed by Wilke et al, ${ }^{22}$ who performed two-stage revision using the VVC implant and reported a mean Knee Society Score (KSS) of
86.6 points at least 10 years postoperatively. Additionally, in the largest study related to VVC implants, Siqueira et $\mathrm{al}^{27}$ showed that the average modified KSS was 72 points in the septic revision group. Although the KSS is not directly comparable to the HSS score, we feel these results are in line with the other one-stage exchange results and are favorable.

\section{Limitations and Strengths}

Inevitably, the current investigation has limitations. Foremost, the design of this retrospective study and the subjective evaluation in terms of the intraoperative soft tissue balance and degree of instability may introduce a selection bias for the patients included in this study. Second, the follow-up period was long enough to clearly identify recurrent or residual infection and early prosthetic failures but may not have been long enough to identify long-term issues with a constrained device. Last, a variety of VVC prostheses with different features were used in the study, depending on the degree of instability, pattern of the bone defects encountered, and the patients' financial state. The various prostheses could not be compared due to limited simple size. However, despite these limitations, we believe our findings are of value, as this study included a relatively uniform patient population and a consistent perioperative protocol and provides the only data regarding midterm survival of VVC implants following one-stage revision for infection.

\section{Conclusion}

This is the first study on the outcomes of one-stage revision using VVC implants for knee PJI. Good midterm survivorship and clinical outcomes are demonstrated at an average of 51.6 months of assessment. Additional follow-ups are required to assess the longterm durability of such implants in the management of instability during the one-stage revision for infected TKA.

\section{Funding}

This study was supported by the following grants: (1) Joint Funds of the National Natural Science Foundation of China, grant nos.: 81760398 and U1503221; (2) National Key Research and Development Program, grant no.: 2017YFB1304204; and (3) Natural Science Foundation of Xinjiang, grant no.: 2021D01C330.

\section{Conflict of Interest}

None declared.

\section{References}

1 Kurtz SM, Lau E, Watson H, Schmier JK, Parvizi J. Economic burden of periprosthetic joint infection in the United States. J Arthroplasty 2012;36(03):61-5.e1

2 Blom AW, Brown J, Taylor AH, Pattison G, Whitehouse S, Bannister GC. Infection after total knee arthroplasty. J Bone Joint Surg Br 2004;86(05):688-691

3 Jämsen E, Huhtala H, Puolakka T, Moilanen T. Risk factors for infection after knee arthroplasty. A register-based analysis of 43,149 cases. J Bone Joint Surg Am 2009;91(01):38-47 
4 Kurtz S, Ong K, Lau E, Mowat F, Halpern M. Projections of primary and revision hip and knee arthroplasty in the United States from 2005 to 2030. J Bone Joint Surg Am 2007;89(04):780-785

5 Insall J, Tria AJ, Scott WN. The total condylar knee prosthesis: the first 5 years. Clin Orthop Relat Res 1979;(145):68-77

6 Röhner E, Benad K, Zippelius T, et al. Good clinical and radiological results of total knee arthroplasty using varus valgus constrained or rotating hinge implants in ligamentous laxity. Knee Surg Sports Traumatol Arthrosc 2019;27(05):1665-1670

7 Lee JK, Lee S, Kim D, et al. Revision total knee arthroplasty with varusvalgus constrained prosthesis versus posterior stabilized prosthesis. Knee Surg Sports Traumatol Arthrosc 2013;21(03):620-628

8 Tibrewal S, Malagelada F, Jeyaseelan L, Posch F, Scott G. Singlestage revision for the infected total knee replacement: results from a single centre. Bone Joint J 2014;96-B(06):759-764

9 Haddad FS, Sukeik M, Alazzawi S. Is single-stage revision according to a strict protocol effective in treatment of chronic knee arthroplasty infections? Clin Orthop Relat Res 2015;473(01):8-14

10 Marx RG. Knee rating scales. Arthroscopy 2003;19(10): 1103-1108

11 McPherson EJ, Woodson C, Holtom P, Roidis N, Shufelt C, Patzakis M. Periprosthetic total hip infection: outcomes using a staging system. Clin Orthop Relat Res 2002;(403):8-15

12 Ji B, Wahafu T, Li G, et al. Single-stage treatment of chronically infected total hip arthroplasty with cementless reconstruction: results in 126 patients with broad inclusion criteria. Bone Joint J 2019;101-B(04):396-402

13 Parvizi J, Zmistowski B, Berbari EF, et al. New definition for periprosthetic joint infection: from the Workgroup of the Musculoskeletal Infection Society. Clin Orthop Relat Res 2011;469 (11):2992-2994

14 Ji B, Li G, Zhang X, Wang Y, Mu W, Cao L. Effective treatment of single-stage revision using intra-articular antibiotic infusion for culture-negative prosthetic joint infection. Bone Joint J 2020;102$\mathrm{B}(03): 336-344$

15 Engh GA, Parks NL. The management of bone defects in revision total knee arthroplasty. Instr Course Lect 1997;46:227-236

16 Gofton WT, Tsigaras H, Butler RA, Patterson JJ, Barrack RL, Rorabeck $\mathrm{CH}$. Revision total knee arthroplasty: fixation with modular stems. Clin Orthop Relat Res 2002;(404):158-168

17 Ji B, Zhang X, Xu B, Guo W, Mu W, Cao L. Single-stage revision for chronic fungal periprosthetic joint infection: an average of 5 years of follow-up. J Arthroplasty 2017;32(08):2523-2530

18 Insall JN, Dorr LD, Scott RD, Scott WN. Rationale of the Knee Society clinical rating system. Clin Orthop Relat Res 1989;(248):13-14

19 Haas SB, Insall JN, Montgomery W III, Windsor RE. Revision total knee arthroplasty with use of modular components with stems inserted without cement. J Bone Joint Surg Am 1995;77(11): 1700-1707

20 Costa GG, Lo Presti M, Agrò G, et al. Difficult primary total knee arthroplasty requiring a varus-valgus constrained implant is at higher risk of periprosthetic infection. Knee Surg Sports Traumatol Arthrosc 2020;28(12):3787-3795

21 Reina N, Salib CG, Pagnano MW, Trousdale RT, Abdel MP, Berry DJ. Varus-valgus constrained implants with a mobile-bearing articulation: results of 367 revision total knee arthroplasties. J Arthroplasty 2020;35(04):1060-1063

22 Wilke B, Wagner E, Trousdale R. Long-term survival of a semiconstrained implant following revision for infection. J Arthroplasty 2015;30(05):808-812

23 Pangaud C, Ollivier M, Argenson JN. Outcome of single-stage versus two-stage exchange for revision knee arthroplasty for chronic periprosthetic infection. EFORT Open Rev 2019;4(08): 495-502

24 Jenny JY, Barbe B, Cazenave A, Roche O, Massin PFrench Society for Hip and Knee Surgery (SFHG) Patient selection does not improve the success rate of infected TKA one stage exchange. Knee 2016; 23(06):1012-1015

25 Massin P, Delory T, Lhotellier L, et al. Infection recurrence factors in one- and two-stage total knee prosthesis exchanges. Knee Surg Sports Traumatol Arthrosc 2016;24(10):3131-3139

26 Anderson JA, Baldini A, MacDonald JH, Tomek I, Pellicci PM, Sculco TP. Constrained condylar knee without stem extensions for difficult primary total knee arthroplasty. J Knee Surg 2007;20(03): 195-198

27 Siqueira MBP, Jacob P, McLaughlin J, et al. The varus-valgus constrained knee implant: survivorship and outcomes. J Knee Surg 2017;30(05):484-492

28 Kim YH, Park JW, Kim JS, Oh HK. Long-term clinical outcomes and survivorship of revision total knee arthroplasty with use of a constrained condylar knee prosthesis. J Arthroplasty 2015;30 (10):1804-1809

29 Breugem SJ, van Ooij B, Haverkamp D, Sierevelt IN, van Dijk CN. No difference in anterior knee pain between a fixed and a mobile posterior stabilized total knee arthroplasty after 7.9 years. Knee Surg Sports Traumatol Arthrosc 2014;22(03):509-516

30 Graves S, Sedrakyan A, Baste V, et al. International comparative evaluation of knee replacement with fixed or mobile-bearing posterior-stabilized prostheses. J Bone Joint Surg Am 2014;96 (Suppl 1):59-64

31 Zeng YM, Yan MN, Li HW, Zhang J, Wang Y. Does mobile-bearing have better flexion and axial rotation than fixed-bearing in total knee arthroplasty? A randomised controlled study based on gait. J Orthop Translat 2019;20:86-93 